\title{
An Algebraic Approach to Synthetic Aperture Sonar Image Reconstruction
}

\author{
Sérgio Rui Silva ${ }^{1}$, Sérgio Cunha ${ }^{1,2}$, Aníbal Matos ${ }^{1}, \mathrm{Nuno} \mathrm{Cruz}^{1}$ \\ ${ }^{1}$ Porto University, Faculty of Engineering (www.fe.up.pt), ${ }^{2}$ CIMAR (www.cimar.org) \\ Email: \{srui, sergio, anibal, nacruz\}@fe.up.pt
}

\begin{abstract}
A new approach for synthetic aperture image formation is presented in this paper. With the presented method image formation is regarded as a signal arrangement that can be described by a matrix. This method integrates the sonar platform motion in the image formation process but more importantly it acknowledges the non ideal data gathering process and implements means to mitigate these shortcomings. This method is illustrated with real data obtained in test mission in the Douro River, Portugal by a synthetic aperture sonar developed at the University of Porto.
\end{abstract}

Keywords- Synthetic aperture, sonar, algebraic image reconstruction, auto-focus

\section{INTRODUCTION}

This paper presents a new approach for synthetic aperture sonar image reconstruction with auto-focus. Starting from the explicit matched filter, it will be shown that it is possible to formulate the image formation process in matrix notation. Furthermore the sonar image will be the solution of the matrix problem that minimizes a criterion that enhances particular characteristics the image (such as signal-to-noise rate, sharpness, etc). This method shares some similarities with blind deconvolution problems typical in the image processing community, and so their particularities are explored to achieve auto-focus ([2]).

With this algorithm one is no longer forced to use or assume a straight line for the sonar platform displacement. The platform deviations from an ideal straight line are not treated as errors, but simply as sampling places ([1]). In the same way, different transducers array geometries are possible without the need for any type of approximation. The navigation information and system geometry is used to build the image formation matrix leading to the reconstructed image. The transmitting and receiving beam patterns and the corresponding swath variation with the platform oscillation is also weighted in the algorithm. This makes this algorithm well suited for high resolution sonar systems with wide swaths and large bandwidths that have the assistance from high precision navigation systems. Only one transducer is needed for image formation and auto-focus, but an array of several elements can also be used to improve image quality and, or, speed of the survey.

The main advantage of this algorithm is the ease of use within an iterative global contrast optimization auto-focus algorithm ([3]). The image formation is divided into two matrixes: a fixed matrix obtained from the sonar geometrical model and navigation data (corresponds to the use of a model matching algorithm, such as the explicit matched filtering); and a matrix of complex adjustable weights that is driven by the auto-focus algorithm. This is valid under the assumption that the image formation matrix is correct at pixel level and the remaining errors are at phase level (so that the complex weight matrix can correct them).

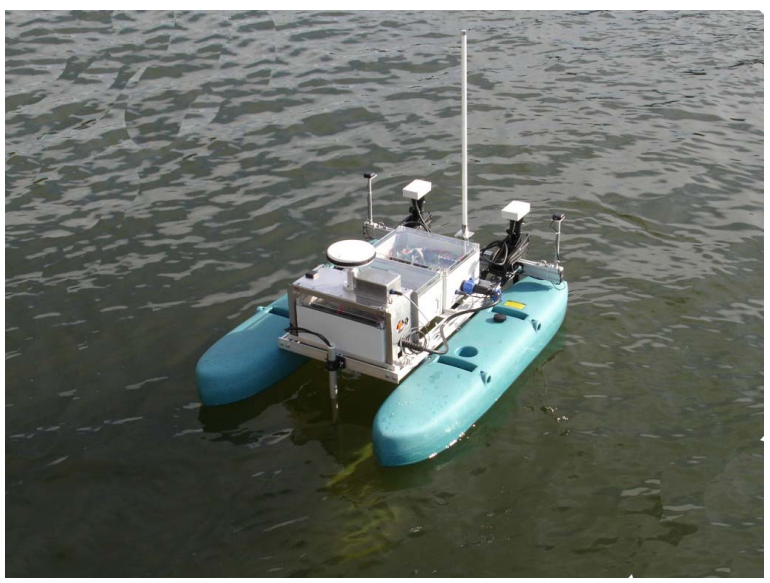

Figure 1: Autonomous boat in operation.

These errors are mainly due to navigation data noise and medium fluctuations and the assumption is valid if such navigation errors are in the order of magnitude of up to one signal wavelength. By putting the image formation process in matrix form, most of the burden of calculation is lightened and the auto-focus algorithm can achieve results in a faster way.

Several methods can be used to update the weight matrix. In this article we will focus on the methods that parameterize the weight matrix and minimize the entropy of the reconstructed image. By doing so, we do not rely in any special feature in the image. Special attention has to be made to local minima and so a suitable optimum search algorithms as to be used.

The image formation matrix can easily grow to a large number of elements (the full matrix would have $(m \times n)^{2}$, were $\mathrm{m} \times \mathrm{n}$ is the number of elements of the resulting image. Nevertheless, the matrix is very sparse and can be dealt with in a column by column or row by row manner. This algorithm is also well suited to be implemented in today's fast graphic processing units since these devices can easily deal with large matrixes and efficiently implement parallel matrix manipulation. 
The algorithm performance is illustrated using real data acquired with a synthetic aperture sonar that is being developed at the University of Porto ([4]). This sonar system is mounted on an autonomous boat that is equipped with a high precision differential GPS system and an inertial navigation unit which, when processed together, produces the navigation data necessary for the presented image reconstruction technique.

This method integrates the sonar platform motion in the image formation process but, moreover, it acknowledges the limitations of the available navigation data and efficiently implements an auto-focus algorithm enabling high quality sonar imagery.

\section{Synthetic Aperture SonAR System Model}

This algorithm was developed for synthetic aperture sonar mounted in a boat but is not in any way restricted to be used in other carriers.

In this case the sonar transducers are located beneath the boat pontoons, rigidly connected to its structure. The boat is programmed to follow a series of straight lines at a constant velocity, during which acoustic signals are sent and the respective echoes are received by the transducers (Figure 2).

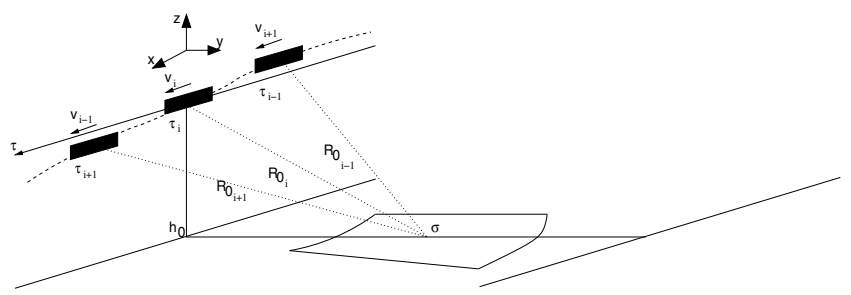

Figure 2: Autonomous boat based SAS model.

Each echo contains indistinctive information of an area corresponding to the radiation pattern of the transducers. The transducers are adjusted so that a swath of about $50 \mathrm{~m}$ is at the boat left broadside. This is known as strip-map configuration.

The echo data, $e e(\tau, t)$, is formed trough the reflection of the transmitted pulse $p_{m}(t)$ on the swath $f f\left(x, y_{s}\right)$ :

$$
e e(\tau, t)=\iint f f\left(x, y_{s}\right) p_{m}\left(t-\frac{2}{c} \sqrt{(v \tau-x)^{2}+y_{s}^{2}}\right) d x d y(1.1)
$$

At each along-track sampling position $\tau$, the echoes caused by the swath reflectivity are received and recorded along $t$.

The task to retrieve the estimated image $f f(x, y)$ is done through the inversion of this model:

$$
f f\left(x, y_{s}\right)=\iint e e(\tau, t) p_{m}\left(t-\frac{2}{c} \sqrt{(v \tau-x)^{2}+y_{s}^{2}}\right) d \tau d t
$$

The sonar platform seldom dislocates through in a straight line, and so the sonar model must account for the irregular along-track sampling positions $([7,12])$. Therefore the polar coordinate model must be abandoned and a broader expression for the echo travel time must contain the estimated position of the transducers and a rough estimation of the bottom height (a flat bottom is enough for a first approximation):

$$
t_{v}(\tau)=\frac{2}{c} \sqrt{\left(x-x_{T}(\tau)\right)^{2}+\left(y-y_{T}(\tau)\right)^{2}+\left(z-z_{T}(\tau)\right)^{2}}
$$

Moreover, different transducer geometries mean that the travel time will not be exactly the simple double of the distance to reach the target, but the distance from the transmitting transducer to the target and back to the receiver which is given by:

$$
t_{v}(\tau)=\frac{1}{c}\left(\left\|T_{T X}^{\prime}(\tau)-X_{\sigma}\right\|+\left\|X_{\sigma}-T_{R X}^{\prime}(\tau)\right\|\right)
$$

Attitude variations must also be accounted for in the calculation of the transducer positions. Knowing the arm between the boat navigation center (reference for the navigation system) and each transducer, and also the roll, pitch and yaw angles, the positions of the transducers can be correctly calculated. The transducers attitude also influences the swath footprint. During image synthesis this information should be used to calculate the correct weights of the combined echoes.

The image formed in this way has a cross-track resolution of $\delta_{X T}=c B W / 2$ and an along-track resolution of $\delta_{A T}=D / 2$ (where $c$ is the speed of sound, $B W$ is the transmitted signal bandwidth and $D$ is the effective transducer diameter). More importantly, the along-track resolution is independent of the target range. To correctly synthesize an image without aliasing artifacts in the along-track dimension, it is necessary to sample the swath with an interval of $D / 4$ (considering the use of only one transducer for transmission and reception). This constraints, together with the maximum PRF defined by the longest distance of interest and the alongtrack sampling restrictions, imposes a very speed to an sonar platform $([10,11])$.

\section{EXPLICIT MATCHED FILTER}

The image formation process of synthetic aperture sonar can be regarded as track and across-track differentiation, by the use of matched filters. The range matched filter is quite simple and obtained by correlating the received echoes with the transmitted pulse (in base-band):

$$
s s_{b}(\tau, t)=e e_{b}(\tau, t) * p_{b}(t)
$$

The range resolution will be given by the signal bandwidth and not by its duration. Because of this, it is possible to use longer pulses to reduce the peak transmitted power, maintaining the total signal energy. The pulse maximum length is limited by the nearest distance of interest.

On the other hand, the along-track compression is more complicated. Several along-track echoes are combined regarding their acquisition positions to form a virtual array which in turn is used to synthesize the image ([1]):

$$
f f\left(x, y_{s}\right)=\iint s s_{b}\left(\tau, t-t_{v}(\tau)\right) e^{-j 2 \pi f_{t_{v}}(\tau)} d \tau d t
$$


Where $f f$ holds the reconstructed image at locations $\left(x, y_{s}\right)$ (slant range and along-track coordinates), $s s_{b}$ is the range-compressed image.

\section{ARITHMETIC RECONSTRUCTION OF SONAR IMAGES}

The integral expression used for the Explicit Matched filter is normally implemented as a summation, due to the discrete nature of the recorded echo data. The samples are interpolated to their correct position given by $t_{v}(\tau)$ and summed to form the synthetic aperture image.

To get a better insight into the algebraic reconstruction algorithm, seeing how interpolation can be implemented in matrix annotation is helpful.

A nearest neighbor interpolation can be written in matrix format in the following way:

$$
\left[\begin{array}{l}
y_{1} \\
y_{2} \\
y_{3} \\
y_{4} \\
y_{5} \\
y_{6}
\end{array}\right]=\left[\begin{array}{llll}
1 & 0 & 0 & 0 \\
0 & 1 & 0 & 0 \\
0 & 1 & 0 & 0 \\
0 & 0 & 1 & 0 \\
0 & 0 & 0 & 1 \\
0 & 0 & 0 & 1
\end{array}\right] \times\left[\begin{array}{l}
x_{1} \\
x_{2} \\
x_{3} \\
x_{4}
\end{array}\right]
$$

Here the samples $\left[y_{1}, y_{2}, y_{3}, y_{4}, y_{5}, y_{6}\right]$ are obtained from $\left[x_{1}, x_{2}, x_{3}, x_{4}\right]$.

The linear interpolation in following a similar manner can also be written in matrix annotation:

$$
\left[\begin{array}{l}
y_{1} \\
y_{2} \\
y_{3} \\
y_{4} \\
y_{5} \\
y_{6}
\end{array}\right]=\left[\begin{array}{cccc}
a_{11} & 0 & 0 & 0 \\
a_{21} & a_{22} & 0 & 0 \\
0 & a_{32} & 0 & 0 \\
0 & 0 & a_{43} & 0 \\
0 & 0 & a_{53} & a_{54} \\
0 & 0 & a_{63} & a_{64}
\end{array}\right] \times\left[\begin{array}{l}
x_{1} \\
x_{2} \\
x_{3} \\
x_{4}
\end{array}\right]
$$

With this in mind, the received echo data can be regarded as a combination of the original image pixels as:

$$
y_{j}=\sum_{i} H_{i j} x_{i}=H_{j} x
$$

Here $x$ is swath reflectivity arranged in vector manner, column by column, and $H_{j}$ a column vector whose non null elements contain the correct complex weights of the abranged swath.

Extending the equation to the complete echo scene we get:

$$
y=H x
$$

Where $\mathrm{y}$ is the echo data image also arranged in a vector, column by column, and $\mathrm{H}$ is a matrix with dimension $(m \times n)^{2}$. Here $m$ is the number of along-track samples and $n$ the number of cross-track samples. This is a very large matrix, but also very sparse.

The reconstructed image can thus be given by:

$$
x=H^{-1} y
$$

Instead of inverting the large matrix $\mathrm{H}$, an approximation can be made which leads to:

$$
x=H^{\prime} y
$$

This is equivalent to the discrete summation that implements the explicit matched filter.

The matrix $\mathrm{H}$ can be regarded as the map of the elements of the image that contributed to one echo. Its elements value and position are calculated through the navigation solution data and transducer parameters as described in the above section. So the navigation data is gracefully included in the image formation process, without any approximation regarding specific paths or transducer location being made. Figure 5 shows the flow diagram of this sonar synthetic aperture processor.

A solution to the above equation can also be obtained using a data fitting method by minimizing iteratively the image $x$ that best fits the model $H$, knowing $y$ :

$$
\min _{x}\|H x-y\|
$$

This, however, does not provide results as good as the autofocus method described in the following section.

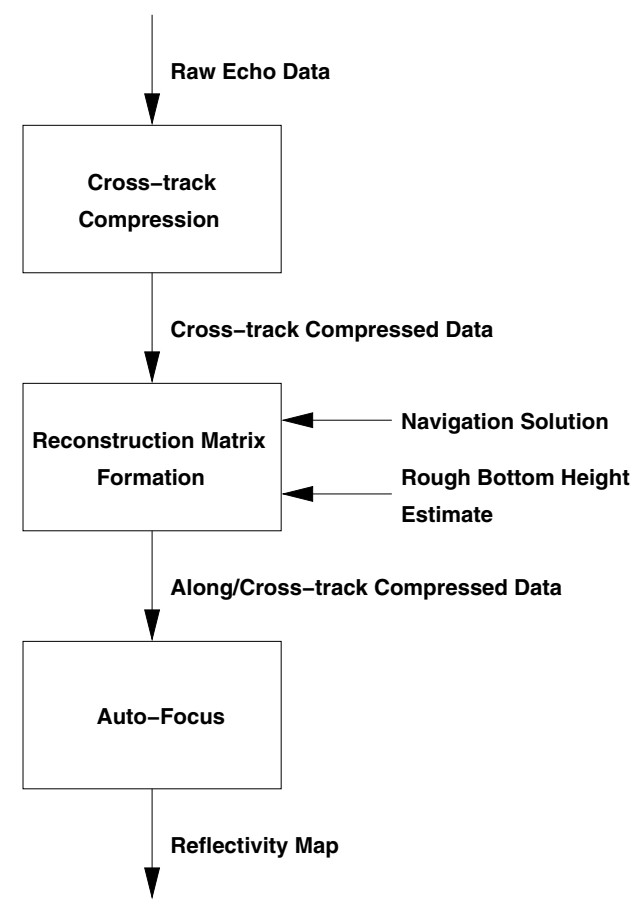

Figure 3: Arithmetic Reconstruction Signal Flow. 


\section{AUTO-Focus}

The use of precise navigation data is fundamental for the reconstruction of synthetic aperture images. Nevertheless, in the case of high frequency sonar systems, it is very difficult to obtain the necessary navigation precision of $1 / 8$ of the wavelength even with the use of an integrated DGPS/INS system.

One of the major advantages of the arithmetic reconstruction algorithm is to lend itself very well to estimation of the correct image formation parameters through the use of a global autofocus algorithm.

Global auto-focus algorithms have several advantages. There is no need for any particular image characteristic such as a strong target or any statistical assumption on the type of clutter. It is also possible to obtain good results with along-track under-sampled data and only one transducer.

For efficient implementation of the auto-focus algorithm, instead of changing the elements of the matrix $H$, which is computationally very demanding, an auxiliary matrix of with complex weights is used:

$$
x=H^{\prime} W y
$$

The matrix $\mathrm{W}$ is diagonal matrix with only $\mathrm{m}$ distinct elements: one each along-track echo. To further simplify this process only the phase of the complex weights is changed.

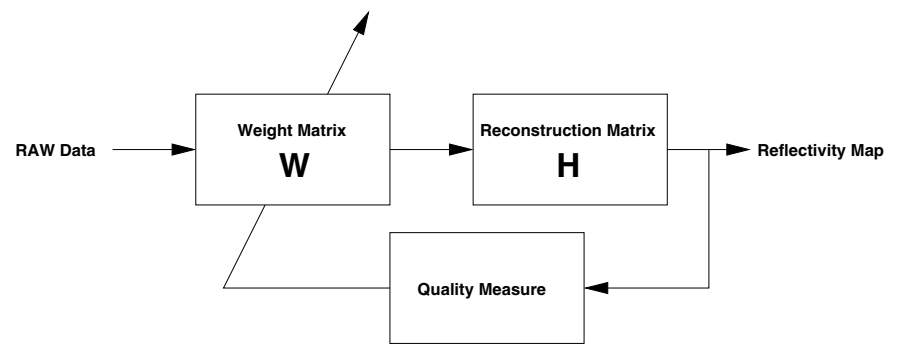

Figure 4: Schematic of the auto-focus procedure.

This naturally assumes that the matrix $\mathrm{H}$ is formed correctly through the use of navigation data to within one wavelength of the transited signal used.

The role of the auto-focus algorithm is thus to search the best set of phase adjustments that maximize some image quality parameter, within one wavelength.

Several image quality measurements can be used, such as contrast or entropy, but it was found that entropy measurement and, in particular, quadratic entropy, is a more robust measure of image quality. The main advantage of quadratic entropy is that no specific assumption of the data probability density function is used. The entropy is calculated using an probability density function estimated through the available data using a Panzer window method as described in [13].

\section{EXTENSION TO A LARGE DATA SET}

Real life data sets might lead to reconstruction matrixes too big to be efficiently computed. In this case, the original echo data can be subdivided into several blocks with suitable overlap. For each block a matrix is created and auto-focus is performed on the respective data. The adjacent blocks use the previous overlapping auto-focus parameters to initialize their own auto-focus step.

\section{RESULTS}

The sonar system is based on an autonomous boat. This is a catamaran like craft (Figure 1) proving high direction stability, smooth operation and several hours of unmanned operation, fulfilling a pre-defined mission plan ([4,8]). Its size is suitable for, together with the navigation system, executing profiles and other maneuvers with sub-meter accuracy. It has two independent thrusters for longitudinal and angular motion providing high maneuverability at low speeds and a maximum speed of $2 \mathrm{~m} / \mathrm{s}$.

The boat communicates with a shore station through a highspeed digital radio-link. This link is used to manually control the boat if needed, to initiate the automatic mission, provide the GPS differential corrections and access the surveyed data in real-time.

This floating platform transports the acoustic transducer array, placed beneath the waterline, a set of GPS a compass and an inertial measurement unit, besides the necessary computational system.

The SAS system is based on a set of simple acoustic transducers and digital signal processing system for signal generation and acquisition ([9]). The transducers operate at a center frequency of $200 \mathrm{kHz}$, corresponding to a wavelength of $0.75 \mathrm{~cm}$. As appropriate for synthetic aperture operations, their real aperture is large (approximately 20 degrees), but have a strong front-to-back lobe ratio which is appropriate to minimize the reflections on the near water surface. The effective transducer diameter is $5 \mathrm{~cm}$, which allows for synthetic images with this order of magnitude of resolution in the along-track direction if suitably focused.. The bandwidth of the signals employed is $30 \mathrm{kHz}$ with the current transducers.

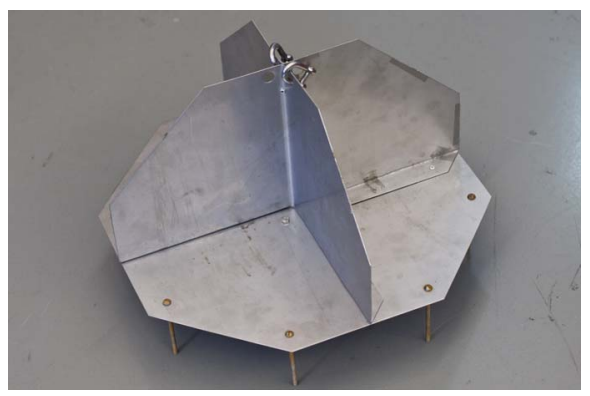

Figure 5: The artificial target used in the test missions. 
To test this synthetic aperture algorithm, an artificial target was placed in the river bottom that served for test site. The target is a half octahedral shape with $20 \times 20 \times 20 \mathrm{~cm}$ (Figure 5). The reflected signal is seen as point after correct image synthesis.
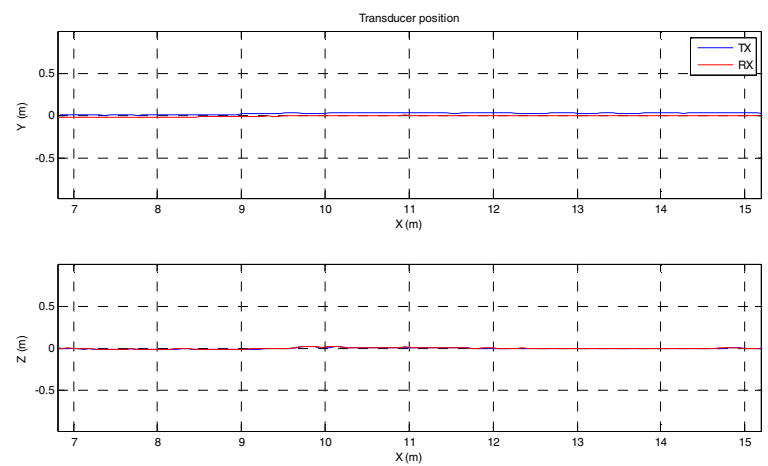

Figure 6: Sonar transducer position.

The boat executed a predefined path (Figure 6) in the vicinity of the target and the swath was sampled along-track at a ratio 4 times lower than the theoretically required.

Figure 7 shows the cross-track compressed image. The hyperbole obtained the strong reflectivity of the artificial target shows little waviness, which is due to the good motion control of the boat.

Figure 8 shows the image synthesized by the arithmetic reconstruction algorithm. Without auto-focus the synthetic aperture reconstruction is not very successful. The error of the navigation system is too high for a successful image synthesis with a signal of this frequency. Although it is possible to distinguish a target, the resolution is poor and the target is smeared in the along track direction.

The mean least square fitting of the data to the reconstruction matrix yields a slightly better (Figure 9), but still not good enough.

Nevertheless using the described auto-focus step, after 25 iterations the image in Figure 10 was obtained. Here the image is perfectly reconstructed. The image quality limitation, in this case, is the side-lobes created by under-sampling in the alongtrack.

As can be seen in Figure 11, which is a contour plot of the magnification of the artificial target image, the resolution is about $2.5 \times 2.5 \mathrm{~cm}$, which is in agreement with the sonar specifications.

Figure 12 shows another passage through the target, now in a position nearer the river margin, trough the several processing steps: cross-track pulse compression, image synthesis trough arithmetic reconstruction and finally image auto-focus.

Again the image without auto-focus is barely perceptible, but after auto-focus the artificial target appears as a sharp point in the image.

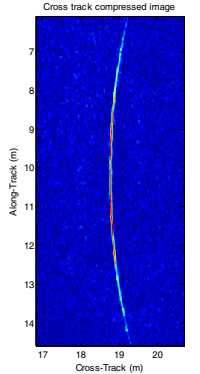

Figure 7: The cross-track compress image.

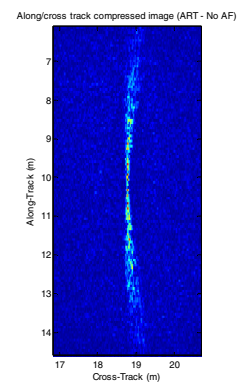

Figure 8: The along/cross-track compressed image without auto-focus.

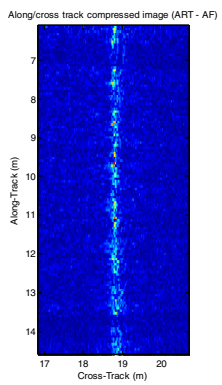

Figure 9: The along/cross-track compressed image min least square fitting.

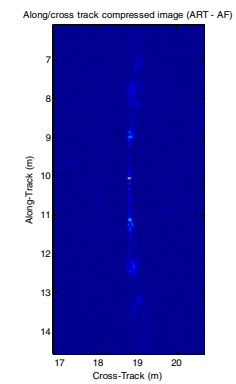

Figure 10: The along/cross-track compressed image with auto-focus. 
For clearer view the image is presented in $3 \mathrm{D}$ in Figure 13. It is possible to see the rope that connected the artificial target to a buoy in the surface in the auto-focused image.

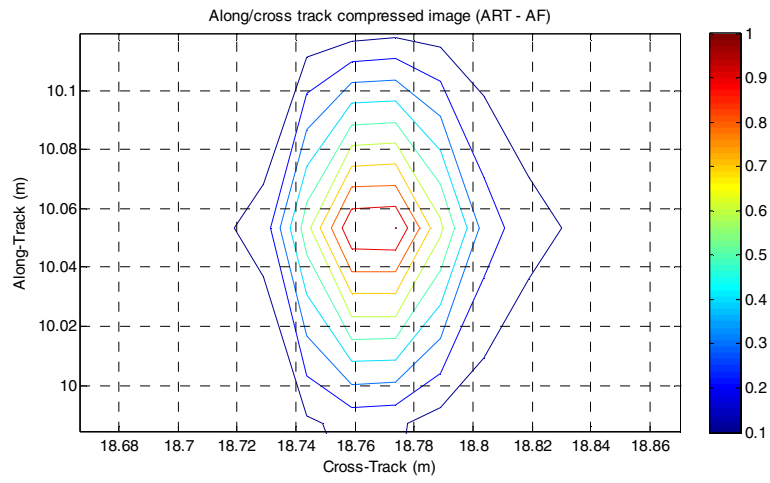

Figure 11: Contour plot of the artificial target image with auto-focus.
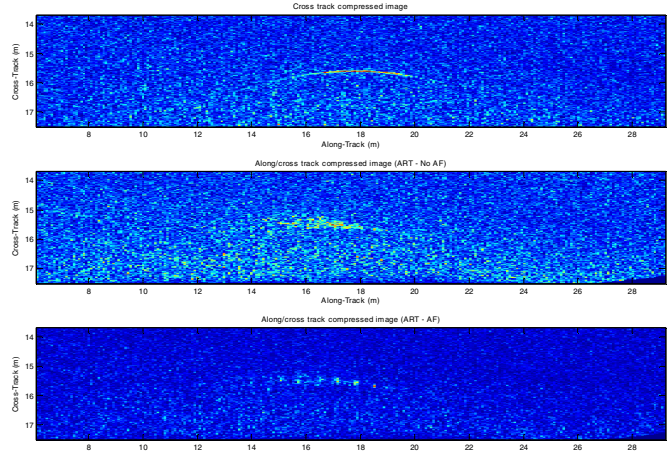

Figure 12: Target with buoy and rope: pulse-compressed, without auto-focus, with auto-focus.

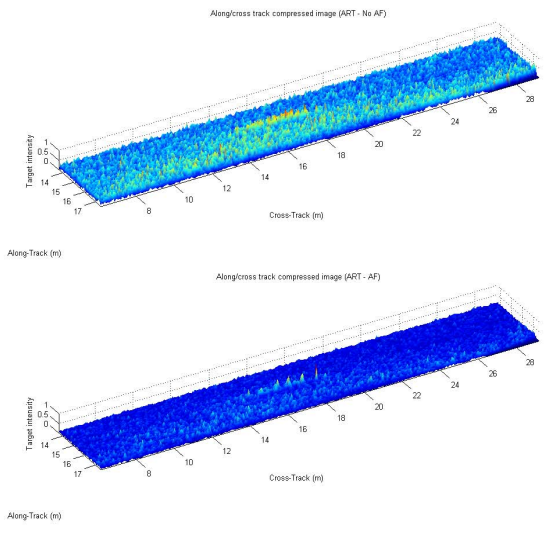

Figure 13: Target with buoy and rope: without auto-focus and with auto-focus.

\section{CONCLUSION}

A new approach to synthetic aperture sonar imaging was presented in this paper. The synthetic aperture is modeled by a matrix that includes navigation information, such as position and attitude, and also the transducers beam aperture. Using this algorithm one is no longer restricted to linear paths, and deviations from this path are not treated as errors, but simply as sampling positions.

Phase errors due to navigation uncertainties and medium fluctuations cause blurring of the image, especially when high frequency signals are used. Nevertheless, the results can be further enhanced through an auto-focus procedure that uses the matrix implementation to efficiently iterate the solutions until convergence to a predefined image quality parameter.

This algorithm enables the acquisition of high quality, high resolution synthetic aperture imagery circumventing some of its problems.

\section{ACKNOWLEDGMENT}

This project has been possible thanks to the contributions of several institutions. The first author is supported by the FCT through the Ph.D. scholarship SFRH/BD/19976/2004/5R38. The authors would like to emphasize the support given by CIIMAR in financing part of the described system.

\section{REFERENCES}

[1] Silkaitis, J.M.; Douglas, B.L.; Hua Lee, "Synthetic-aperture sonar imaging: system analysis, image formation, and motion compensation," Signals, Systems and Computers, 1995. 1995 Conference Record of the Twenty-Ninth Asilomar Conference on , vol.1, no., pp.423-427 vol.1, 30 Oct-1 Nov 1995.

[2] Kundur, D.; Hatzinakos, D., "Blind image deconvolution," Signal Processing Magazine, IEEE, vol.13, no.3, pp.43-64, May 1996.

[3] Fortune, S.A.; Hayes, M.P.; Gough, P.T., "Statistical autofocus of synthetic aperture sonar images using image contrast optimisation," OCEANS, 2001. MTS/IEEE Conference and Exhibition , vol.1, no., pp.163-169 vol.1, 2001

[4] Silva, S.R.; Cunha, S.; Matos, A.; Cruz, N., "An Autonomous Boat Based Synthetic Aperture Sonar," Oceans 2007 , vol., no., pp.1-7, Sept. 29 2007-Oct. 42007.

[5] P. T. Gough, "Unified Framework for Modern Synthetic Aperture Imaging Algorithms". The International Journal of Imaging Systems and Technology, Vol. 8, pp. 343-358.

[6] Silkaitis, J.M.; Douglas, B.L.; Hua Lee, "Synthetic-aperture sonar imaging: system analysis, image formation, and motion compensation," Signals, Systems and Computers, 1995. 1995 Conference Record of the Twenty-Ninth Asilomar Conference on , vol.1, no., pp.423-427 vol.1, 30 Oct-1 Nov 1995.

[7] Shippey, G.; Banks, S.; Pihl, J., "SAS image reconstruction using Fast Polar Back Projection: comparisons with Fast Factored Back Projection and Fourier-domain imaging," Oceans 2005 - Europe , vol.1, no., pp. 96101 Vol. 1, 20-23 June 2005.

[8] N. Cruz, A. Matos, S. Cunha, S. Silva, "ZARCO - An Autonomous Craft for Underwater Surveys", Proceedings of the 7th Geomatic Week, Barcelona, Spain, Feb 2007.

[9] S. Silva, S. Cunha, A. Matos, N. Cruz, "An In-SAS System For Shallow Water Surveying", Proceedings of the 7th Geomatic Week, Barcelona, Spain, Feb 2007.

[10] McHugh, R.; Shaw, S.; Taylor, N., "Spatial sampling ambiguities in synthetic aperture sonar for broadside and squint look operations," OCEANS '98 Conference Proceedings, vol.2, no., pp.960-964 vol.2, 28 Sep-1 Oct 1998.

[11] Tomiyasu, K., "Tutorial review of synthetic-aperture radar (SAR) with applications to imaging of the ocean surface," Proceedings of the IEEE, vol.66, no.5, pp. 563-583, May 1978. 
[12] Fornaro, G., "Trajectory deviations in airborne SAR: analysis and compensation ," Aerospace and Electronic Systems, IEEE Transactions on, vol.35, no.3, pp.997-1009, Jul 1999.
[13] W. Liu, P. P. Pokharel, J. C. Principe, "Correntropy: A Localized Similarity Measure", International Joint Conference on Neural Networks, 2006, pp. 4919 - 4924 